

\title{
Experimental and numerical investigations of low-temperature superfluid turbulence
}

Malek Abid, Marc Brachet, Jean Maurer, Caroline Nore, Patrick Tabeling

\section{To cite this version:}

Malek Abid, Marc Brachet, Jean Maurer, Caroline Nore, Patrick Tabeling. Experimental and numerical investigations of low-temperature superfluid turbulence. European Journal of Mechanics B/Fluids, 1998, 17 (4), pp.665-675. 10.1016/S0997-7546(98)80019-8 . hal-00426545

\section{HAL Id: hal-00426545 https://hal.science/hal-00426545}

Submitted on 29 Mar 2021

HAL is a multi-disciplinary open access archive for the deposit and dissemination of scientific research documents, whether they are published or not. The documents may come from teaching and research institutions in France or abroad, or from public or private research centers.
L'archive ouverte pluridisciplinaire HAL, est destinée au dépôt et à la diffusion de documents scientifiques de niveau recherche, publiés ou non, émanant des établissements d'enseignement et de recherche français ou étrangers, des laboratoires publics ou privés.

\section{(c)(1)}

Distributed under a Creative Commons Attribution| 4.0 International License 


\title{
Experimental and numerical investigations of low-temperature superfluid turbulence
}

\author{
M. ABID ${ }^{a *}$, M. E. BRACHET ${ }^{b}$, J. MAURER ${ }^{b}$, C. NORE $^{c}$, P. TABELING $^{b}$
}

\begin{abstract}
Low-temperature superfluid turbulence is studied experimentally in a Helium swirling flow and numerically with the GrossPitaevskii equation in the geometry of the Taylor-Green (TG) vortex flow. Numerically, it was found in Nore et al. (1997a, b) that the kinetic energy transfer in the superfluid TG vortex is comparable to that of the viscous TG vortex and that the energy spectrum of the superflow is compatible with Kolmogorov's scaling. The vorticity dynamics of the superflow are similar to that of the viscous flow. In both cases, many vortex reconnection events happen throughout the flow. Experimentally, power measurements and pressure fluctuation spectra show very little difference above and far below the superfluid transition temperature, where the normal-fluid component of Helium is negligible (less than 5\% in mass at $T=1.2 \mathrm{~K}$ ).
\end{abstract}

\section{Introduction}

Helium $\mathrm{He}^{4}$, below the $\lambda$ transition, behaves as a superposition of two fluids (Landau and Lifchitz, 1980): a superfluid and a normal fluid. The normal-fluid component is a classical fluid with viscosity $\mu_{n}$. The superfluid component contains vortex filaments with an atomic scale core radius, $\xi \sim 1 \AA$, and a quantized circulation $\Gamma=h / m_{\mathrm{H}^{p}}=0.99 \times 10^{-7} \mathrm{~m}^{2} \mathrm{~s}^{-1}$. Each fluid has its own density $\rho_{n}$ and $\rho_{s}$ and velocity field $\mathbf{v}_{n}$ and $\mathbf{v}_{s}$ (the subscripts refer to the normal fluid and superfluid). The two fluids interact through mutual friction caused by the interaction of the normal fluid with the superfluid vortex filaments.

Experiments and simulations (Schwarz, 1985) with the supcrfluid and the normal fluid flowing in opposite directions (defined as "counterflow" and produced by a heat current) show that the superfluid vortex filaments form an intricate tangle with dissipation due to the relative velocity between the two fluids. This behavior has no classical analog. In contrast, recent results with superfluid and normal fluid flowing in the same direction ("coflow") as in towed grid superfluid turbulence (Smith et al., 1993) were interpreted as the flow of a single fluid of density $\rho=\rho_{n}+\rho_{s}$ and viscosity $\mu_{n}$ (Samuels, 1992). Because of mutual friction, the filaments follow the flow imposed by the normal-fluid component. Note that a non-negligible proportion of normal-fluid component is necessary for this kind of interpretation.

The nature of coflow superfluid turbulence, when the temperature is low enough for the normal-fluid component to be neglected (in practice below $1.4 \mathrm{~K}$ ), is the main subject of the present paper. We study this regime (which will be simply called "superfluid turbulence" in the rest of the paper) by comparing the results of numerical simulations and experiments. The numerical simulations are performed using the Gross-Pitaevskii equation

\footnotetext{
"Institut de Recherche sur les Phénomènes Hors Équilibre, UMR CNRS et Université d'Aix-Marseille I, service 252, Centre Saint-Jérôme, 13397 Marseille Cedex 20, France

${ }^{b}$ Laboratoire de Physique Statistique de l'École Normale Supérieure, associé au CNRS et aux Universités Paris 6 et 7, 24, rue Lhomond, 75231 Paris Cedex 05, France

'Laboratoire d'Informatique pour la Mécanique et les Sciences de l'Ingénieur, BP133, 91403 Orsay Cedex, France

* Correspondence and reprints
} 
(Gross, 1963; Ginzburg, 1958), also called the Nonlinear Schrödinger Equation (NLSE), which is an adequate mathematical description of superflows in the low temperature regime.

This paper is organized as follows: in the first part, we will briefly recall some recent numerical results (Nore et al., 1977a, b) based on high resolution simulations of NLSE; in the second part, we will present new experimental results performed in a Helium swirling flow above and well below the transition temperature $T_{\lambda} \sim 2.2 \mathrm{~K}$. Finally, we will discuss the physical mechanisms involved in low-temperature superfluid turbulence.

\section{Numerical results using the Gross-Pitaevskii equation}

The Gross-Pitaevskii equation or NLSE is the partial differential equation for a complex wave field $\psi$ :

$$
\partial_{t} \psi=i c /(\sqrt{2} \xi)\left(\psi-|\psi|^{2} \psi+\xi^{2} \nabla^{2} \psi\right)
$$

where $c$ and $\xi$ are respectively the sound velocity and the so-called "coherence length". The mass flux is proportional to $i(\bar{\psi} \nabla \psi-\psi \nabla \bar{\psi})$ and the density to $|\psi|^{2}$ ( $\bar{\psi}$ is the complcx conjugate of $\psi$ ). The compressible superlow is irrotational, except on the nodal lines of the complex wave field. These lines are known to follow Eulerian dynamics. They are non-singular, in contrast to the singular vortex lines in Landau's model (Landau and Lifchitz, 1980). These topological defects correspond to the quantum vortices of superfluid Helium; they appear naturally - with the correct amount of velocity circulation - in this model. In this context, NLSE is the correct dynamic equation of motion for superfluids (Nozières, 1990).

We have qualified the degree of analogy between turbulence in low-temperature superfluids and incompressible viscous fluids (Nore et al., 1997a, b) by comparing numerical simulations of NLSE with existing numerical simulations of the Navier-Stokes equations, in the Taylor-Green (TG) vortex (Taylor and Green, 1937). The viscous TG vortex is defined as the solution of the Navier-Stokes equations with periodic boundary conditions and initial data $\mathbf{v}^{\mathrm{TG}}=(\sin (x) \cos (y) \cos (z),-\cos (x) \sin (y) \cos (z), 0)$. The TG flow is closely related to an experimental swirling flow (Douady et al., 1991; Zocchi et al., 1994; Fauve et al., 1993). The experimental flow and the TG vortex have similar geometries (Douady et al., 1991): a shear layer between two counterrotating eddies. The TG vortex, however, is periodic in contrast to the experimental flow which is contained inside a tank between two counter-rotating disks.

Two technical tools, necessary for a study of turbulence in numerical solutions of NLSE, were developed in Nore et al. $(1997 \mathrm{a}, \mathrm{b})$. The first tool generates initial data, for NLSE that will reproduce the vorticity dynamics, without too much sound wave emission, of any given 3D incompressible flow field, provided that this field admits a Clebsch potential representation (which is the case for the TG initial velocity $\mathbf{v}^{\mathrm{TG}}$ (Nore et al., 1997a)).

The second numerical tool uses the expression for the total energy density in physical space and Parseval's formula to define energy spectra; these make possible a separation of the various contributions to the total energy.

One of the main quantitative results obtained by Nore et al. $(1997 \mathrm{a}, \mathrm{b})$ is the remarkable agreement of the energy dissipation rate with the corresponding data in the incompressible viscous TG flow. Both the moment $t_{\max } \sim 10$ of maximum energy dissipation and its value $\varepsilon\left(t_{\max }\right) \sim 10^{-2}$ at that moment are in quantitative agreement with the viscous data (Brachet et al., 1983) (see Fig. 1). Furthermore, it can be seen in Figure 1 that the dissipation is rather insensitive to the value of the coherence length $\xi$.

Another important quantity studied in turbulence is the scaling of the kinetic energy spectrum. Figure 2 (b) shows that, in the interval $2 \leq k \leq 16$, the spectrum of incompressible kinetic energy is compatible with the $k^{-5 / 3}$ Kolmogorov scaling. The same scaling is also displayed on Figure 2 (a) to show that Kolmogorov's scaling does not hold at short times but is obtained at later times after the building-up of energy at intermediate 


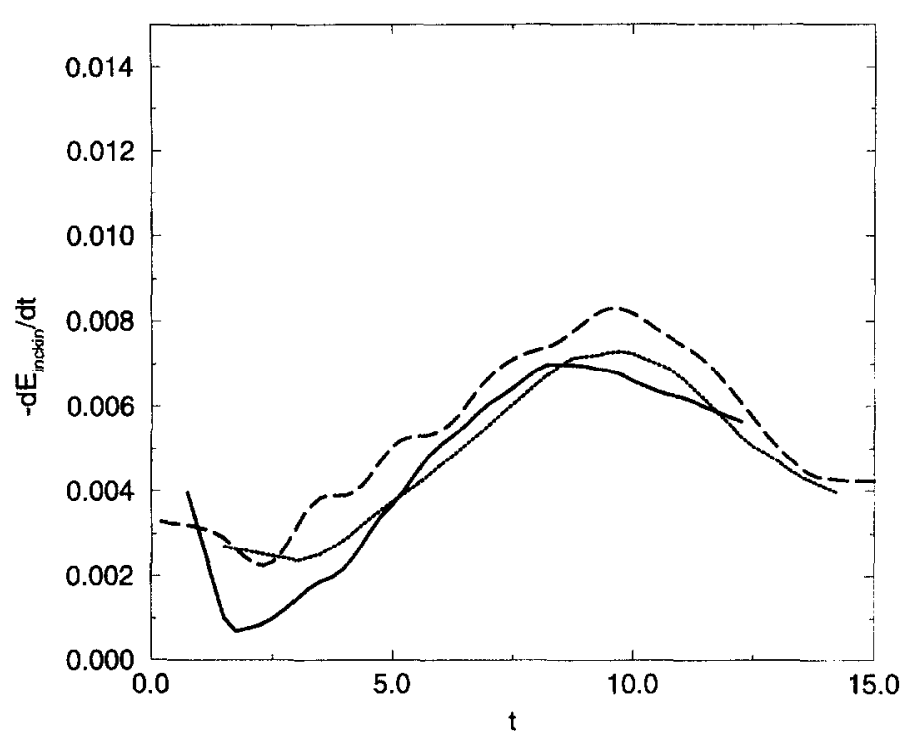

Fig. 1. - Evolution of the total incompressible kinetic energy dissipation $-d E_{k i n}^{i} / d t$ for: run $a$ with $\xi=0.1 /(4 \sqrt{2})$ and $N=256$ (long-dashed), run $b$ with $\xi=0.1 /(6.25 \sqrt{2})$ and $N=400$ (dotted) and run $c$ with $\xi=0.1 /(8 \sqrt{2})$ and $N=512$ (solid), where $N$ is the grid resolution. All runs are realised with $c=2$.

scales $2 \leq k \leq 16$. The wavenumber $k=16$ corresponds to the averaged distance between vortex lines $l_{b}$ (see below in the discussion section).

The vortex lines of the superfluid TG vortex are visualized in physical space in Figures 3, 4 and 5 at times $t=0, t=5$ and $t=9$. At $t=5$, a few reconnections have taken place while a complex vortex tangle is present at $t=9$. Note that, in the viscous TG vortex, reconnection also sets in for $t \geq 5$.

The evolution of the total vortex filament length in the $512^{3}$ run (see caption of Fig. 1) is displayed in Figure 6 where it can be seen that the total length has increased by a factor of 3 at the end of the run.

These numerical results have lead us to conjecture the existence of a Kolmogorov regime in experimental low temperature superflows (Nore et al., 1977a, b).

\section{Experimental results below and above $T_{\lambda}$}

\section{A. DESCRIPTION OF THE EXPERIMENT}

The experimental set-up we use is similar to the one described in Maurer et al. (1994). Actually, some modifications have been made to work in a lower range of temperatures, extending down to $1.2 \mathrm{~K}$. The flow is produced in a cylinder, $8 \mathrm{~cm}$ in diameter and $12 \mathrm{~cm}$ high, limited axially by two counter-rotating disks. One disk is flat and, on the other one, are fixed 8 radial blades, making an angle of $45^{\circ}$ between each other. Each blade is an aluminium plate, $1 \mathrm{~mm}$ thick, $2.5 \mathrm{~cm}$ wide and $4 \mathrm{~cm}$ long mounted normally to the plane of the disk. A stator is mounted at half the total height of the cell in order to stabilize the turbulent shear region. The two disks are driven by two DC motors rotating from 1 to $30 \mathrm{~Hz}$. The whole system is enclosed directly in a liquid Helium bath which is used as the experimental fluid. This is the main difference with the set-up described in Maurer et al. (1994). We now use liquid Helium as the working fluid, in a range extending from $1.2 \mathrm{~K}$ to $4.2 \mathrm{~K}$; the temperature of the fluid is fixed by the pressure above the liquid bath, which is itself controlled by the pumping system we use. Temperature is measured by means of an Allen Bradley resistor immersed in the liquid bath. 

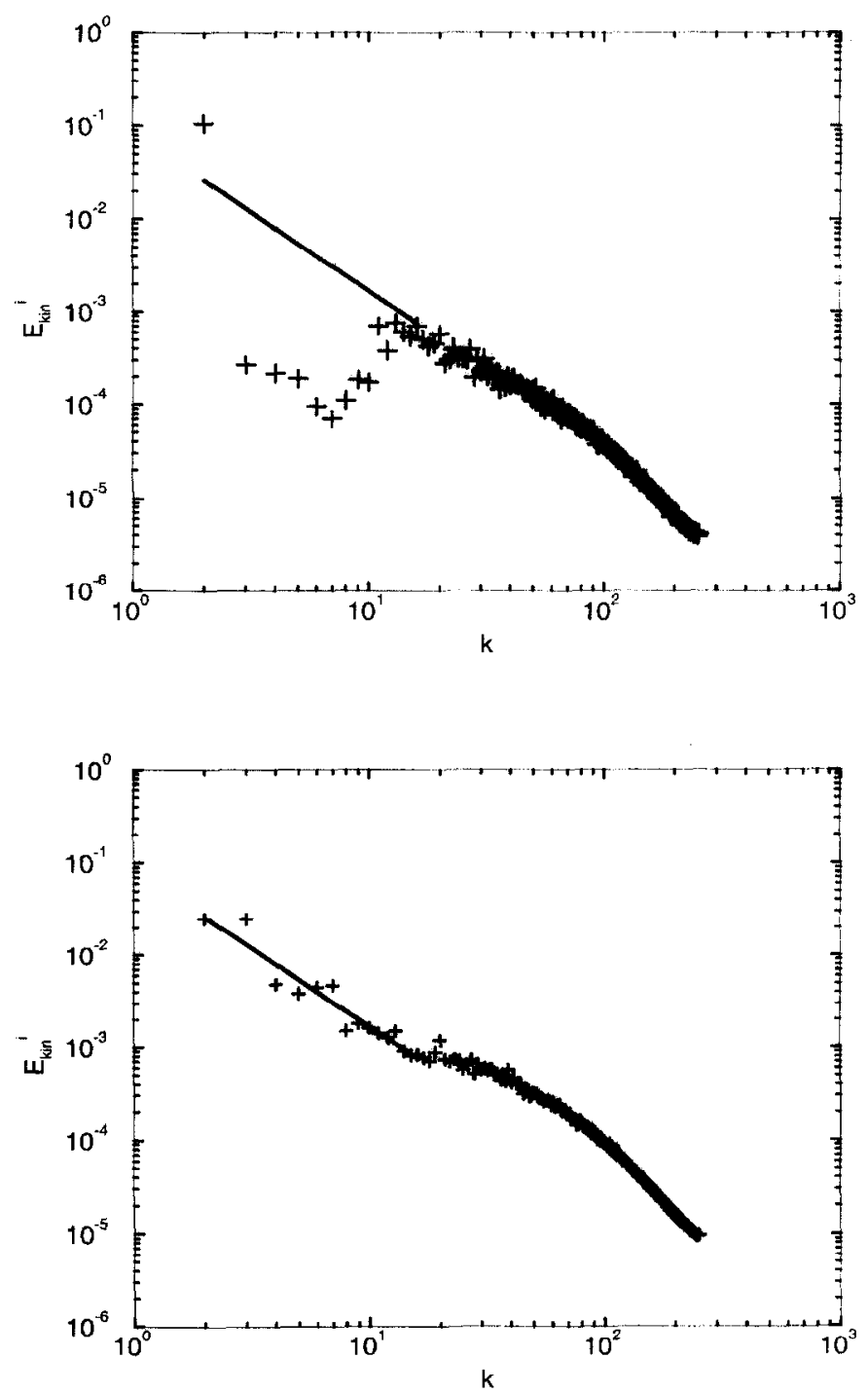

Fig. 2. - Incompressible kinetic energy spectrum at $t=0$ (a) and $t=5.5$ (b). A fit with power law $C k^{-n}$ in the range $2 \leq k \leq 16$ gives, at $t=5.5, n=1.70$ (b). The same power law is also displayed in the upper figure (a) for comparison (see text).

\section{B. Measured quantities}

Measuring local velocity in superfluid turbulence is a challenge; standard hot wire anemometry does not seem to work in superfluid Helium because the thermal transfers in the working fluid are much more efficient than in viscous Helium, and thus it is difficult to hold a substantial temperature difference between the sensor and the ambient fluid. We have nonetheless obtained information of dynamical significance by measuring, for a prescribed velocity, and at various temperatures, a quantity proportional to the torque exerted by the fluid on the disks. The quantity at hand is the electrical current supplying the DC motors since for the regimes we consider - we have checked this point independently - the mechanical and electrical losses can be neglected.

Local pressure fluctuations are also measured by using small total-head pressure tubes, immersed in the flow. The pressure sensors are hollow metallic tubes, connected to a quartz pressure transducer WHM 112 A22 from $\mathrm{PCB}$. Various dimensions for the inner and outer tube diameters have been tested, in a range varying from 0.3 




Fig. 3. - Three-dimensional visualization of the vorticity field for the Taylor-Green flow at time $t=0$. The visualization is obtained by drawing the 30000 vectors of highest norm in the $[0, \pi] \times[0, \pi] \times[0, \pi]$ box.

to $1 \mathrm{~mm}$ for the inner diameter and from 0.6 to $3 \mathrm{~mm}$ for the outer one. The tip of the probe is directed against the mean flow. The sensor, albeit rather large compared to the usual sizes of hot-wire anemometers, nonetheless allows to resolve one decade of scale below the injection scale, which is enough to address the inertial range. The local pressure fluctuations are conveyed along the inner part of the tube, and transmitted, through a small chamber, to a low impedance quartz pressure transducer. Details are given in Maurer et al. (1998).

In normal fluids, the pressure measured at the tip of the total-head tube can be related to the upstream flow $U(t)$ and the local pressure $P(t)$ by using the Bernoulli theorem, provided several assumptions are made. The assumptions are that the flow scales we address are much larger than the tube diameter, the viscosity is negligible (i.e. the Reynolds number based on the probe diameter is large), and the instantaneous flow pattern around the probe is quasi-steady. In such circumstances, one gets:

$$
P_{\text {meas }}(t)=P(t)+\rho U^{2}(t) / 2 \text {. }
$$

In the flow region where the probe is immersed, a well established axial mean flow $U$ exists so that, after removing the mean parts of Equation (2), one gets:

$$
p_{\text {meas }}(t)=p(t)+\rho U u(t)
$$




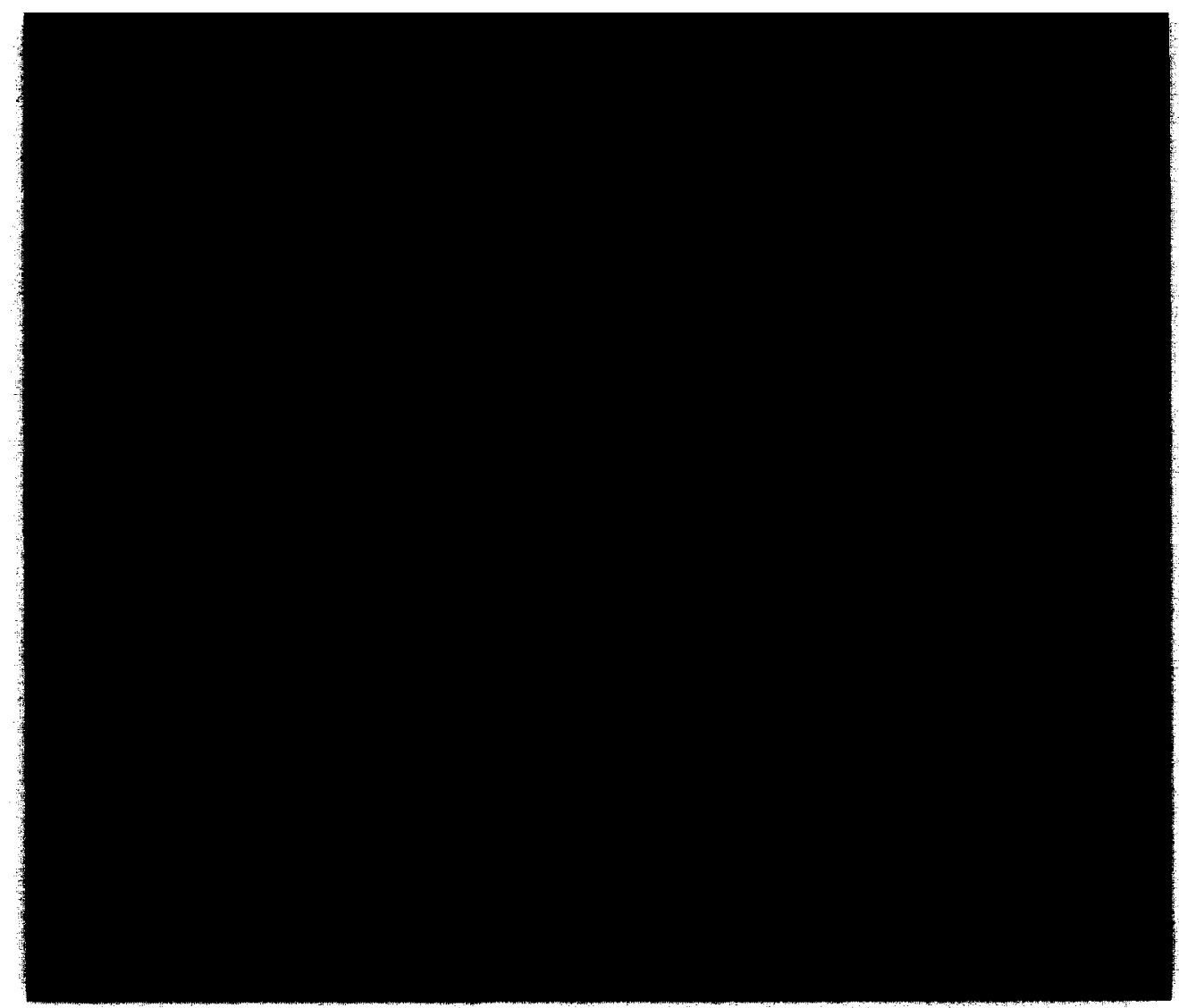

Fig. 4. - Same visualization as in Figure 3 but at time $t=5$. A few reconnections have occurred.

where now $p_{\text {meas }}, p$ and $u$ are the fluctuations of the measured pressure, the actual pressure, and the local velocity respectively. It is currently admitted that, in ordinary turbulent situations, and at low fluctuation rates, Equation (3) is dominated by the dynamic term, so that by measuring the pressure fluctuations at the total head tube, one has a direct access to the velocity fluctuations $u(t)$.

The situation is less clear when the probe is immersed in the superfluid. It is however possible to write an equation similar to (3) in the context of the Landau two-fluid model, by assuming the densities $\rho_{s}$ and $\rho_{n}$ of the superfluid and normal components are constant, the system is isentropic, the viscosity of the normal fluid is negligible, and the streamlines developing around the total head tube, both for the superfluid and the normal components, are close to those of a stationary potential flow; under such conditions, one gets instead of (3), the following relation:

$$
P_{\text {meas }}(t)=P(t)+\rho_{s} U_{s}^{2}(t) / 2+\rho_{n} U_{n}^{2}(t) / 2
$$

in which $U_{s}(t)$ and $U_{n}(t)$ are the velocities of the superfluid and normal phases respectively. In the case where the probe is in a region of vigorous mean flow, characterized by mean velocities $U_{s}$ and $U_{n}$ one has, similarly to (3):

$$
p_{\text {meas }}(t)=p(t)+\rho_{s} U_{s} u_{s}(t)+\rho_{n} U_{n} u_{n}(t)
$$




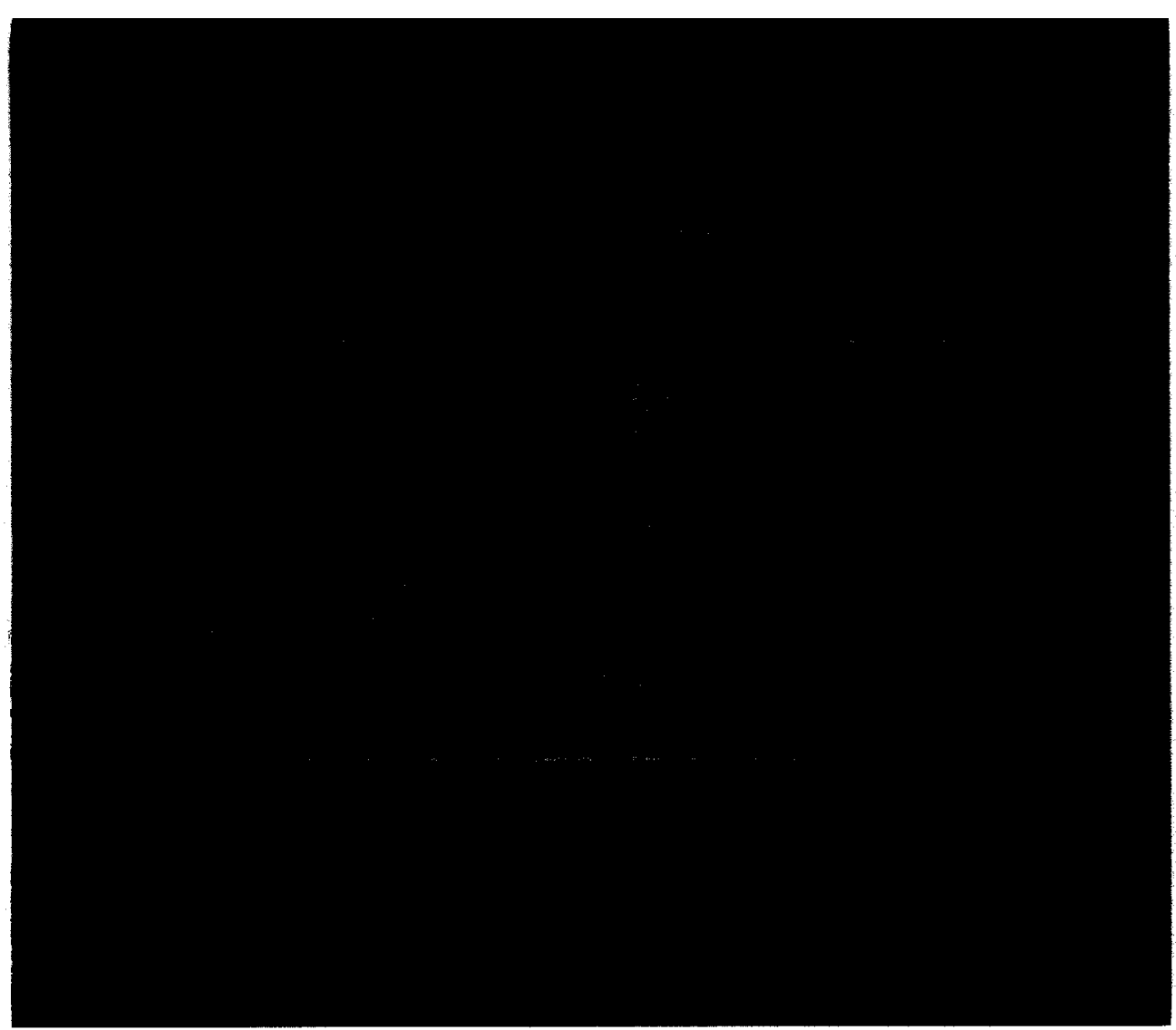

Fig. 5. Same visualization as in Figure 3 but at time $t=9$. Many reconnections have produced an intricate turbulent vortex filament tangle.

where now $p_{\text {meas }}, p, u_{s}$, and $u_{n}$ are the fluctuations of the measured pressure, the actual one, and the velocities of the superfluid and normal flow components. Rclation (5) indicates that a total head pressure tube immersed in Helium II measures a linear combination of the pressure and normal and superfluid velocity component fluctuations. It is difficult to simplify relation (5) further at this stage, except perhaps close to $T_{\lambda}$ where the normal component is dominant compared with the superfluid one and far below $T_{\lambda}$ where we have the opposite situation.

\section{RESULTS}

Figure 7 shows, for a rotation rale of the disks equal to $4 \mathrm{~Hz}$ (bladed) and $8 \mathrm{~Hz}$ (flat), the temperature dependence of the time averaged electrical current supplying the DC motors.

For both types of disks, the typical velocity within the fluid is of the order of $40 \mathrm{~cm} / \mathrm{s}$, and, above $T_{\lambda}$, the overall Reynolds number (based on the disk angular velocity and the disk radius) is of the order of $10^{6}$. On the same plot, we represent, below $T_{\lambda}$, the concentration of superfluid Helium. The striking observation is that, for a given rotation speed, the electrical currents (and therefore the torques applied on the disks) do not vary with temperature. This result is easy to interpret above $T_{\lambda}$, since it is known that when normal fluids are used, and for disks equipped with blades, the torque does not depend on viscosity (Cadot et al., 1997). In our situation, it is remarkable to observe that as we penetrate into the superfluid region, the torque remains a constant even in the extreme case where the superfluid Helium phase forms $95 \%$ of the working fluid. The case without blades 


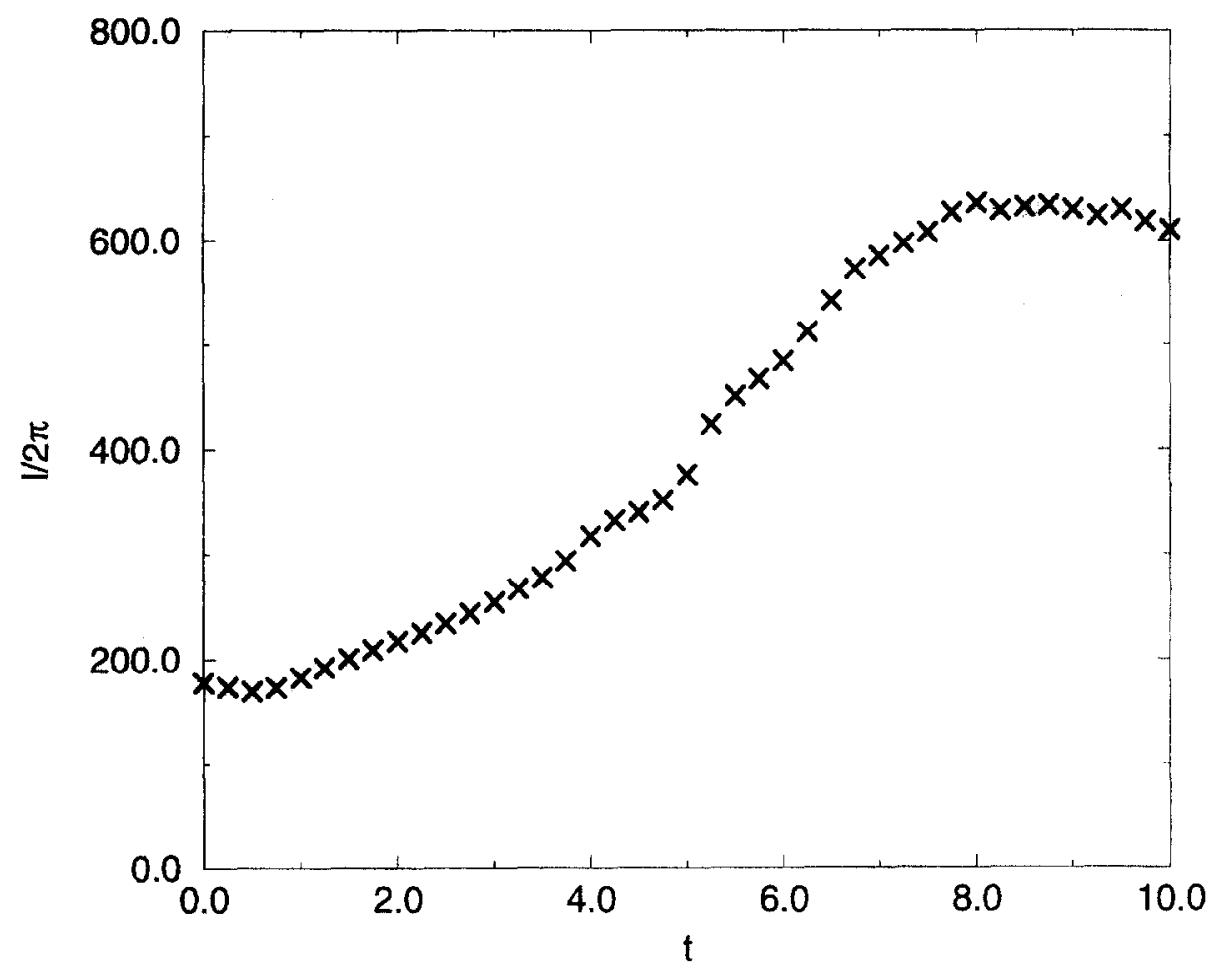

Fig. 6. - Vortex filament length divided by $2 \pi$ in run $c$ with $\xi=0.1 /(8 \sqrt{2})$ and resolution $N=512$. Because of vortex stretching, the total length has increased by a factor 3 .



Fig. 7. - Electric current (in $m A$ ) feeding the DC motors as a function of temperature. Circles: flat disk (motor driven at $2 \mathrm{~V}$ ), diamonds: bladed disk (motor driven at $1 \mathrm{~V}$ ). The solid line shows the proportion of superfluid (right $y$ axis). 
is somewhat more delicate to interpret, since experiments carried out in normal fluids have not shown that, in a way similar to boundary layers, the torque depends on the fluid viscosity. In our case however, estimates suggest that roughness of the disks is above the viscous sublayer so that viscosity effects, above $T_{\lambda}$, may be unimportant. The constancy of the torque below $T_{\lambda}$, as displayed in Figure 7 for the disk without blades, suggests that the same line of argument holds even in the superfluid region. However, a full interpretation of these results probably requires an understanding of the interaction of superfluids with walls in the turbulent regime.

The analysis of the pressure fluctuations obtained with the total head tube placed at $2 \mathrm{~cm}$ above the mid plane and $2 \mathrm{~cm}$ from the cylinder axis, yields interesting information. Figures 8 and 9 show the spectra of the pressure fluctuations above and below $T_{\lambda}$ (i.e. respectively at $2.3 \mathrm{~K}$ and $1.4 \mathrm{~K}$ ). Figure 8 clearly shows, as expected, that



Fig. 8. - Experimental pressure fluctuation spectrum (in non-dimensional units) measured with a total head pressure tube immersed in the flow at $T=2.3 \mathrm{~K}$.

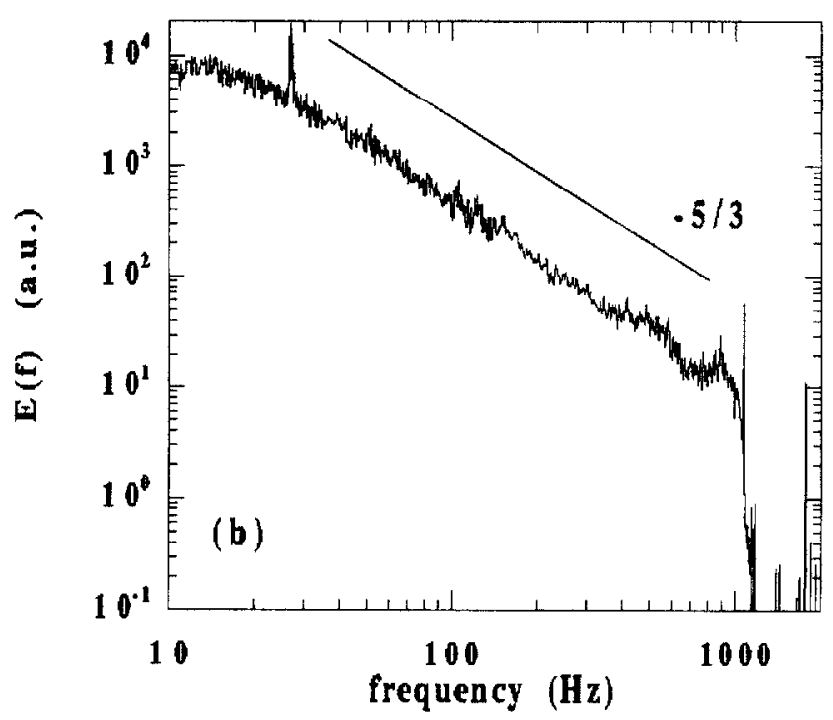

Fig. 9. - Experimental pressure fluctuation spectrum (in non-dimensional units) measured with a total head pressure tube immersed in the flow at $T=1.4 \mathrm{~K}$. 
such fluctuations follow a Kolmogorov regime between the injection scale (signalled by the peak at $25 \mathrm{~Hz}$ ) and the largest frequency we can resolve, i.e. $900 \mathrm{~Hz}$. The spectrum obtained at $1.4 \mathrm{~K}$ is similar to that of Figure 9 . We clearly get a Kolmogorov-like regime in the same range of frequencies. The corresponding Kolmogorov constant turns out also to be indistinguishable from the classical value. We have further analyzed the deviations from Kolmogorov in the superfluid regime. The striking result is that they have the same magnitude as in classical turbulence. More details are given in Maurer et al., 1998).

These observations - both on global and local quantities - agree pretty well with the theoretical approach developed in the first part of the paper. In particular, it seems rather clear that Kolmogorov cascade survives in the superfluid regime. The experiment moreover shows that the deviations from the Kolmogorov regime also survive, with the same magnitude. This point might be interesting to investigate numerically, in the framework discussed in Section II.

\section{Discussion}

The results presented here are consistent with the existence of a Kolmogorov regime in low-temperature superfluid turbulence.

It thus appears that low-temperature superfluid turbulence behaves in a way that is very similar to viscous turbulence. Some physical mechanisms are common to the superfulid and viscous cases while others are completely different.

The prominent common mechanisms are: (i) the existence of vortex lines following Eulerian dynamics at large scale (large radius of curvature); (ii) the possibility, when small enough scales are reached, to reconnect the vortex lines.

While both mechanisms are present in the viscous and superfluid cases, their physical nature is dramatically different. Superfluid vortex lines are discrete, with a quantized circulation $\Gamma$, while the viscous vorticity is continuous. In the superfluid case, they reconnect naturally as nodal lines in a nonlinear wave equation while viscous vortex lines reconnect by diffusion.

A tangle of superfluid vortex lines can mimic the behavior of viscous turbulence with continuous vorticity by giving rise to a local averaged vorticity that can be compared to the viscous vorticity. This is possible because three different length-scales are present in the superfluid problem (Nore et al., 1997b). The largest scales are the energy injection (integral) length and velocity scales $l_{I}$ and $u_{I}$. The smallest scale is the vortex core size $\xi$ (of the order of $1 \AA$ in Helium). An intermediate scale is given by the averaged distance between vortex lines $l_{l}$, (see Figs. 2 (a), 2 (b) and Fig. 3). It can be shown (Nore et al., 1997b) that $l_{b}=l_{I} \sqrt{\Gamma / l_{I} u_{I}}$, where $\Gamma=h / m_{\mathrm{H}}$. is the quantum of circulation. In a regime where $l_{I} \gg l_{b} \gg \xi$, the comparison with viscous turbulence can be made in the range of scales $l_{I}>l>l_{b}$. Note that, in the experiment reported in this article, $l_{I} \sim 1 \mathrm{~cm}, l_{b} \sim 100$ $\mu \mathrm{m}$ and $\xi \sim 1 \AA$. The physical mechanism in superfluid turbulence that takes place at scales $l<l_{b}$ cannot be compared to viscous diffusion. However it is a result of our numerical simulations that the incompressible kinetic energy that reaches these scales is transferred into a bath of compressible sound waves. Experimentally, this corresponds to phonon excitations. If the temperature is low enough, the ballistic phonons will reach the boundaries of the experiment. If the temperature is high enough to have substantial normal fluid, the phonons will locally increase the normal-fluid density $\rho_{n}$. Thus the energy is finally dissipated in a thermodynamically irreversible way, just as in viscous turbulence. 


\section{REFERENCES}

Brachet M. E., Meiron D. I., Orszag S. A., Nickel G., Morf R. H., Frisch U., 1983, Small-scale structure of the Taylor-Green vortex, J. Fluid Mech., 130, 411-452.

Cadot O., Couder Y., Daerr A., Douady S., Tsinober A., 1997, Energy injection in closed turbulent flows: stirring through boundary layers versus inertial strirring, Phys. Rev. E, 56, 427-433.

DOUADY S., COUdER Y., BRACHET M. E., 1991, Direct observation of the intermittency of intense vorticity filaments in turbulence, Phys. Rev. Lett., 67, 983-986.

Fauve S., Laroche C., Castaing B., 1993, Pressure fluctuations in swirling turbulent flows, J. Phys. II, 3, $271-278$.

Ginzburg V. L., Pitaevskil L. P., 1958, On the theory of superfluidity, Sov. Phys. JETP, 34, 858-861.

Gross E. P., 1963, Hydrodynamics of a Superfluid Condensate, J. Math. Phys., 4, 195-207.

Landau L., LifChiTZ E., 1980, Fluid Mechanics, Pergamon Press, Oxford.

Maurer J., TABeling P., 1998, Local investigation of superfluid turbulence, Europhys. Lett, accepted.

Maurer J., Tabeling P., ZocChi G., 1994, Statistics of Turbulence between two counterrotating Disks in Low-temperature Helium Gas, Europhys. Lett., 26, 31-36.

NORE C., ABID M., BRACHET M., 1997a, Decaying Kolmogorov turbulence in a model of superflow, Phys. Fluids, 9, 2644-2669.

Nore C., ABID M., BRACHET M., 1997b, Kolmogorov turbulence in low-temperature superflows, Phys. Rev. Lett., 78, $3896-3899$.

Nozières P., PINES D., 1990, The Theory of Quantum Liquids, Addison Wesley, New York.

SAmuel.S D. C., 1993, Respones of superfluid vortex filaments to concentrated normal-fluid vorticity, Phys. Rev. B, 47, $1107-1110$.

Scliwarz K. W., 1985, Three-dimensional vortex dynamics in supcrfluid ${ }^{4} \mathrm{Hc}$ : linc-line and linc-boundary interactions, Phys. Rev. B, 31, $5782-5804$.

Smith M., Donnelly R., Goldenfeld N., Vinen W., 1993, Decay of vorticity in homogeneous turbulence, Phys. Rev. Lett., 71, $2583-2586$.

TAylor G. I., Green A. E., 1937, Mechanism of the production of small eddies from large ones, Proc. Roy. Soc. Lond. A, 158, 499-521.

Zocchi G., Tabeling P., Maurer J., Willaime H., 1994, Measurement of the scaling of the dissipation at high Reynolds numbers, Phys. Rev. E, 50, 3693-3700. 